\title{
Conformally de Sitter space from anisotropic SD3-brane of type IIB string theory
}

\author{
Shibaji Roy 1 \\ Saha Institute of Nuclear Physics \\ 1/AF Bidhannagar, Calcutta 700064, India
}

\begin{abstract}
We construct a four dimensional de Sitter space upto a conformal transformation by compactifying the anisotropic SD3-brane solution of type IIB string theory on a six dimensional product space of the form $H_{5} \times S^{1}$, where $H_{5}$ is a five dimensional hyperbolic space and $S^{1}$ is a circle. The radius of the hyperbolic space is chosen to be constant. The radius of the circle and the dilaton in four dimensions are time dependent and not constant in general. By different choices of parameters characterizing the SD3-brane solution either the dilaton or the radius of the circle can be made constant but not both. The form field is also non vanishing in general, but it can be made to vanish without affecting the solution. This construction might be useful for a better understanding of dS/CFT correspondence as well as for cosmology.
\end{abstract}

\footnotetext{
${ }^{1}$ E-mail: shibaji.roy@saha.ac.in
} 
Space-like branes [1,2] or S-branes, for short, are interesting time dependent Euclidean brane solutions that exist in many field theories as well as string/M theory. These are soliton-like objects localized in space-time and therefore exist only for a moment in time. In string theory, S-branes arise as the time dependent solution of world-volume tachyon (or the rolling tachyon) of the non-BPS D-brane or D-brane/anti-D-brane pair [3, 4]. The original motivation for studying S-branes was to understand the holography in the temporal context. In particular, as the spatial holography or AdS/CFT gives us an additional space-like direction from a Lorentzian field theory, the temporal holography can similarly give a time-like direction from an Euclidean field theory which is necessary for the dS/CFT correspondence [5]. The space-time construction of these S-branes was thus motivated to understand the proposed dS/CFT correspondence.

Even though much work has been done on S-branes, the original motivation how to relate these objects to de Sitter space remained unclear. In this Letter we give a simple construction of four dimensional de Sitter space upto a conformal transformation from certain S-brane solution of type IIB string theory. This not only clarifies the relation between S-branes and de Sitter space, but also could be useful in the cosmological context. It is well-known that our universe is going through an accelerated expansion [6] in the present epoch [7,8] and S-branes [1,9 12] are known to give rise to such an accelerated expansion [13 17] but the acceleration in that context is found to be transient with efolding of the order of unity. But if S-brane can be related conformally to de Sitter space, we can get a realistic cosmology in that particular conformal frame. Of course, the significance of that conformal frame, which is not clear to us at present, needs to be understood.

We must mention that despite the no-go theorem of Maldacena-Nunez [18], many metastable de Sitter vacua have been found (circumventing the no-go theorem) in the literature from Type IIB, IIA and heterotic string theory starting from the original work by Kachru-Kallosh-Linde-Trivedi [19] by the so-called geometric/non-geometric flux compactification [20,21]. This approach also solves another important problem of fixing the moduli. However, explicit classical solutions have not been reported in the literature. In this Letter we will construct one such classical solution, which is not exactly de Sitter but conformal to it, from type IIB string theory. To be precise, we start from the known [22, 23] non-supersymmetric configuration of intersecting charged D3-brane with chargeless D0-brane solution of type IIB string theory and take a double Wick rotation to obtain the SD3-brane solution, which is anisotropic in one spatial direction. (Note that the S-brane here is called SD-brane because it possesses the same charge as the D-brane.) This solution is characterized by four independent parameters. We then write the solution 
in a convenient coordinate and take a 'near horizon' limit after fixing one of the parameters. The resultant solution can then be compactified on a five dimensional hyperbolic space [24 26] of constant radius and a circle of time dependent radius (in general) to obtain a four dimensional space, which can be shown to be conformal to a one-parameter family (the other two parameters can be set to some convenient values without any loss of generality) of de Sitter space. In this solution the dilaton is non-constant. The free parameter of the solution can be chosen in appropriate ways to make either the radius of $\mathrm{S}^{1}$ or the dilaton constant. But both of them can not be made constant simultaneously. The form-field also is non-zero in general, but it can be put to zero without affecting the solution.

The non-supersymmetric charged Dp-brane intersecting with chargeless D0-brane solution of type II string theory is given in [22,23]. For $p=3$, it is a type IIB string theory solution and is given by,

$$
\begin{aligned}
& d s^{2}=-F^{-\frac{1}{2}}\left(\frac{H}{\tilde{H}}\right)^{\frac{3 \delta_{1}}{8}+\frac{3}{2} \delta_{0}} d t^{2}+F^{-\frac{1}{2}}\left(\frac{H}{\tilde{H}}\right)^{-\frac{5 \delta_{1}}{8}-\frac{1}{2} \delta_{0}} \sum_{i=1}^{3}\left(d x^{i}\right)^{2} \\
& +F^{\frac{1}{2}}(H \tilde{H})^{\frac{1}{2}}\left(\frac{H}{\tilde{H}}\right)^{\frac{3 \delta_{1}}{8}}\left(d r^{2}+r^{2} d \Omega_{5}^{2}\right) \\
& e^{2\left(\phi-\phi_{0}\right)}=\left(\frac{H}{\tilde{H}}\right)^{\frac{\delta_{1}}{2}-6 \delta_{0}}, \quad F_{5}=(1+*) Q \operatorname{Vol}\left(\Omega_{5}\right)
\end{aligned}
$$

where the various functions appearing in the above solution are defined as,

$$
\begin{aligned}
H & =1+\frac{\omega^{4}}{r^{4}}, \quad \tilde{H}=1-\frac{\omega^{4}}{r^{4}} \\
F & =\left(\frac{H}{\tilde{H}}\right)^{\alpha} \cosh ^{2} \theta-\left(\frac{\tilde{H}}{H}\right)^{\beta} \sinh ^{2} \theta .
\end{aligned}
$$

In (11) $\phi$ is the dilaton field and $\phi_{0}$ is its asymptotic value. $F_{5}$ is the self-dual five form, where $Q$ is the charge parameter and $*$ denotes the Hodge dual. The solution is characterized by seven parameters, namely, $\alpha, \beta, \delta_{0}, \delta_{1}, \omega, \theta$, and $Q$. The equations of motion give us three relations among them and they are

$$
\begin{aligned}
& \alpha-\beta=-\frac{3}{2} \delta_{1} \\
& \frac{1}{2} \delta_{1}^{2}+\frac{1}{2} \alpha\left(\alpha+\frac{3}{2} \delta_{1}\right)+\frac{3}{2} \delta_{0}^{2}=\left(1-3 \delta_{0}^{2}\right) \frac{5}{4} \\
& Q=4 \omega^{4}(\alpha+\beta) \sinh 2 \theta .
\end{aligned}
$$

Therefore, there are actually four independent parameters in the solution. Now we can get the anisotropic space-like D3 brane or SD3-brane solution from the above solution (1) 
by the double Wick rotation $r \rightarrow i t, t \rightarrow-i x^{4}$, alongwith $\omega \rightarrow i \omega, \theta_{1} \rightarrow i \theta_{1}$ (where $\theta_{1}$ is one of the angles of the sphere $\left.\Omega_{5}\right)$ and $\theta \rightarrow i \theta$. The solution (1) then takes the form,

$$
\begin{aligned}
d s^{2}=F^{\frac{1}{2}}(H \tilde{H})^{\frac{1}{2}}\left(\frac{H}{\tilde{H}}\right)^{\frac{3 \delta_{1}}{8}}(- & \left.d t^{2}+t^{2} d H_{5}^{2}\right)+F^{-\frac{1}{2}}\left(\frac{H}{\tilde{H}}\right)^{\frac{3 \delta_{1}}{8}+\frac{3}{2} \delta_{0}}\left(d x^{4}\right)^{2} \\
& +F^{-\frac{1}{2}}\left(\frac{H}{\tilde{H}}\right)^{-\frac{5 \delta_{1}}{8}-\frac{1}{2} \delta_{0}} \sum_{i=1}^{3}\left(d x^{i}\right)^{2} \\
e^{2\left(\phi-\phi_{0}\right)}=\left(\frac{H}{\tilde{H}}\right)^{\frac{\delta_{1}}{2}-6 \delta_{0}}, \quad F_{5}= & -(1+*) Q \operatorname{Vol}\left(H_{5}\right),
\end{aligned}
$$

where the various functions are now defined as,

$$
\begin{aligned}
H & =1+\frac{\omega^{4}}{t^{4}}, \quad \tilde{H}=1-\frac{\omega^{4}}{t^{4}} \\
F & =\left(\frac{H}{\tilde{H}}\right)^{\alpha} \cos ^{2} \theta+\left(\frac{\tilde{H}}{H}\right)^{\beta} \sin ^{2} \theta .
\end{aligned}
$$

The parameter relations (3) remain exactly the same except the last one, which gets modified as $Q=4 \omega^{4}(\alpha+\beta) \sin 2 \theta$. Eq.(4) represents the anisotropic SD3-brane solution which has a four dimensional Euclidean world-volume with one anisotropic direction $x^{4}$. Also note that the solution is well-defined as long as $t>\omega$.

We now make a coordinate transformation,

$$
t=\tau\left(\frac{1+\sqrt{g(\tau)}}{2}\right)^{\frac{1}{2}}, \quad \text { where } \quad g(\tau)=1+\frac{4 \omega^{4}}{\tau^{4}} \equiv 1+\frac{\tau_{0}^{4}}{\tau^{4}}
$$

In terms of this new coordinate the metric and the dilaton in (4) take the forms,

$$
\begin{aligned}
& d s^{2}=G^{\frac{1}{2}} g^{\frac{\alpha}{4}+\frac{1}{4}+\frac{3 \delta_{1}}{16}}\left(-\frac{d \tau^{2}}{g}+\right.\left.\tau^{2} d H_{5}^{2}\right)+G^{-\frac{1}{2}} g^{-\frac{\alpha}{4}+\frac{3 \delta_{1}}{16}+\frac{3 \delta_{0}}{4}}\left(d x^{4}\right)^{2} \\
&+G^{-\frac{1}{2}} g^{-\frac{\alpha}{4}-\frac{5 \delta_{1}}{16}-\frac{\delta_{0}}{4}} \sum_{i=1}^{3}\left(d x^{i}\right)^{2} \\
& e^{2\left(\phi-\phi_{0}\right)}=g^{\frac{\delta_{1}}{4}-3 \delta_{0}}, \quad \text { where, } \quad G=\cos ^{2} \theta+g^{-1} \sin ^{2} \theta .
\end{aligned}
$$

In writing the function $G$, we have put $\alpha+\beta=2$ for simplicity as we will see. We also have from (3),$\alpha-\beta=-(3 / 2) \delta_{1}$ and so, combining these two we get, $\alpha=1-(3 / 4) \delta_{1}$. Using this in the second parameter relation, given in (3), we get

$$
\frac{7}{8} \delta_{1}^{2}+21 \delta_{0}^{2}=3
$$


Now we take the 'near horizon' limit $\tau \ll \tau_{0}$ and in that case $g(\tau)=1+\tau_{0}^{4} / \tau^{4} \approx \tau_{0}^{4} / \tau^{4}$ and $G=\cos ^{2} \theta+g^{-1} \sin ^{2} \theta \approx \cos ^{2} \theta$. With these the metric and the dilaton reduce to,

$$
\begin{aligned}
& d s^{2}=-\frac{\cos \theta \tau^{2}}{\tau_{0}^{2}} d \tau^{2}+\frac{\tau_{0}^{3 \gamma-1}}{\cos \theta \tau^{3 \gamma-1}}\left(d x^{4}\right)^{2}+\frac{\tau^{\gamma+1}}{\cos \theta \tau_{0}^{\gamma+1}} \sum_{i=1}^{3}\left(d x^{i}\right)^{2}+\cos \theta \tau_{0}^{2} d H_{5}^{2} \\
& e^{2\left(\phi-\phi_{0}\right)}=\frac{\tau_{0}^{\delta_{1}-12 \delta_{0}}}{\tau^{\delta_{1}-12 \delta_{0}}}, \quad \text { where } \quad \gamma=\frac{1}{2} \delta_{1}+\delta_{0} .
\end{aligned}
$$

Note that here we have obtained the radius of the five dimensional hyperbolic space to be constant given by $\tau_{0} \sqrt{\cos \theta}$. This happened because we had chosen the condition $\alpha+\beta=2$. If we did not use this condition the radius would have been time dependent. This simplifies the compactification on hyperbolic space. Further, for simplification let us put $\tau_{0}=1$ and $\cos \theta=1$. Note that $\cos \theta=1$ implies $Q=0$ and therefore the form-field is absent. Again this is not necessary, but we use this just for simplification. Now with these simplifications we can compactify the metric successively on $H_{5}$ and $x^{4}$ (notice that $x^{4}$ has a time dependent radius in general when $3 \gamma-1 \neq 0$ ), i.e., on a product space of the type $H_{5} \times S^{1}$ and the resultant four dimensional metric in the Einstein frame as well as the dilaton can be written as,

$$
\begin{aligned}
& d s_{4}^{2}=\eta^{3}\left[-\frac{4}{(\gamma-3)^{2}} \frac{d \eta^{2}}{\eta^{2}}+\frac{\sum_{i=1}^{3}\left(d x^{i}\right)^{2}}{\eta^{2}}\right] \\
& e^{2\left(\phi-\phi_{0}\right)}=\eta^{\frac{2\left(\delta_{1}-12 \delta_{0}\right)}{\gamma-3}},
\end{aligned}
$$

where we have defined a new time coordinate by the relation $\tau^{\gamma-3}=\eta^{-2}$. We recognize the metric in (10) to be a one-parameter family of four dimensional de Sitter metric upto a conformal factor $\eta^{3}$. One may think that since we can freely choose the parameter $\left(\delta_{1}\right.$ or $\delta_{0}$ related by the relation (8) ), we can use the freedom to make the metric purely de Sitter, but that is not possible. The reason is, since $\delta_{1}$ and $\delta_{0}$ have to be real, they can not take arbitrary values. In fact, we see from (8) that we must have $0 \leq\left|\delta_{1}\right| \leq 2 \sqrt{6} / \sqrt{7}$, such that both $\delta_{0}$ and $\delta_{1}$ remain real. Similarly, we should have $0 \leq\left|\delta_{0}\right| \leq 1 / \sqrt{7}$, such that the parameters remain real. Because of this restriction, we can not have a de Sitter metric from SD3-brane. Note that the constant factor in front of $-d \eta^{2} / \eta^{2}$ term can be scaled away by scaling $\eta$ and $x^{1,2,3}$ appropriately. Also, by using the parameter relation (8) we can easily check that the maximum value of $\gamma=(1 / 2) \delta_{1}+\delta_{0}$ is +1 and the minimum value is -1 . The maximum of $\gamma$ occurs when $\delta_{1}=12 / 7$ and $\delta_{0}=1 / 7$ and the minimum occurs when $\delta_{1}=-12 / 7$ and $\delta_{0}=-1 / 7$. At these two extremal values the exponent of $\eta$ in the dilaton expression given in (10) vanishes and the dilaton becomes constant. The metric also simplifies at these two extremal values of $\gamma$. Thus, it is clear that the de 
Sitter metric upto a conformal factor can actually be obtained from pure gravity in ten dimensions without the dilaton and the form-field. Also note that the radius of $x^{4}$ can be made constant by putting $\gamma=(1 / 2) \delta_{1}+\delta_{0}=1 / 3$. The value of $\delta_{0}$ and $\delta_{1}$ in that case can be determined from (8) to be, $\delta_{0} \simeq 0.38$ or, -0.28 and $\delta_{1} \simeq-0.09$ or, 1.23. But, with these values of the parameters the dilaton would be time dependent. We would like to mention that we could have relaxed the condition $\alpha+\beta=2$ and in that case radius of the hyperbolic space would also become time dependent as we mentioned before and one might think that this would give more freedom to choose the parameters and the compactification might lead to purely de Sitter space in four dimensions. But, again we have checked that this is not possible. The parameters are restricted in such a way that we can obtain de Sitter only upto a conformal factor. Finally, we would like to point out that in order for the supergravity description to remain valid in various regimes of time, the radius of the circle $S^{1}$ must be large compared to the string scale, otherwise, we have to go to the T-dual frame (or type IIA theory) and the effective string coupling $e^{2 \phi}$ must remain small compared to $e^{2 \phi_{0}}=g_{s}^{2}$ (where $g_{s}$ is the string coupling), otherwise, we have to go to the S-dual frame (of type IIB theory).

To understand the significance of de Sitter space upto a conformal transformation in string theory SD3-branes, we point out that this is analogous to the near horizon limit of $\mathrm{D} p$-branes of type II string theories for $p \neq 3,5$, where AdS spaces show up upto a conformal transformation. The implication of the appearance of conformally AdS spaces in the latter case is that, there exists a more general correspondence, than AdS/CFT, which has been named as domain wall/QFT correspondence [27. In fact, it has been shown in [27], that there exists a special conformal frame (or dual frame) in which the 10dimensional supergravity action leads to a $p+2$-dimensional gauged supergravity action upon compactification on $\mathrm{S}^{8-p}$. The corresponding Einstein frame action then admits an $\mathrm{AdS}_{p+2}$ 'linear dilaton' vacuum which is equivalent to the domain wall solution in this conformal frame. One is thus led to the conjecture that the QFT describing the internal dynamics of large $N$ number of coincident $\mathrm{D} p$-branes is equivalent to string theory on domain wall background [27]. For the case of conformally de Sitter space in SD3-brane solution we expect an Euclideanized version of domain wall/QFT-like correspondence to hold. It would be interesting to substantiate this claim with more supporting evidence, for example, comparing the symmetries and also to see whether similar correspondences hold for other SD-branes of type II string theories.

Thus, in this Letter, we have given a simple construction of a four dimensional de Sitter space upto a conformal factor by compactifying the anisotropic SD3-brane solution of type IIB string theory on a six dimensional internal space of the form $H_{5} \times S^{1}$. The 
radius of $H_{5}$ is chosen to be constant. The radius of $S^{1}$ and the dilaton are in general time dependent. However, they can be made constant separately by appropriate choices of parameters. This brings out the connection between space-like branes and the de Sitter space which might be useful to understand dS/CFT correspondence better in the same spirit as AdS/CFT. Our construction may also be useful in the context of cosmology, as looking from a particular conformal frame, we obtain a four dimensional universe with a positive cosmological constant, consistent with observation.

Acknowledgements: I am grateful to J. X. Lu for very useful comments and discussions. I would also like to thank Amit Ghosh and Harvendra Singh for useful discussions.

\section{References}

[1] M. Gutperle and A. Strominger, JHEP 0204, 018 (2002) hep-th/0202210.

[2] A. Maloney, A. Strominger and X. Yin, JHEP 0310, 048 (2003) hep-th/0302146.

[3] A. Sen, hep-th/9904207.

[4] A. Sen, JHEP 0204, 048 (2002) hep-th/0203211.

[5] A. Strominger, JHEP 0110, 034 (2001) hep-th/0106113.

[6] A. G. Riess et al. [Supernova Search Team Collaboration], Astrophys. J. 560, 49 (2001) astro-ph/0104455.

[7] A. Lewis and S. Bridle, Phys. Rev. D 66, 103511 (2002) astro-ph/0205436.

[8] C. L. Bennett et al. [WMAP Collaboration], Astrophys. J. Suppl. 148, 1 (2003) astro-ph/0302207.

[9] C. -M. Chen, D. V. Gal'tsov and M. Gutperle, Phys. Rev. D 66, 024043 (2002) hep-th/0204071.

[10] M. Kruczenski, R. C. Myers and A. W. Peet, JHEP 0205, 039 (2002) hep-th/0204144.

[11] S. Roy, JHEP 0208, 025 (2002) hep-th/0205198.

[12] S. Bhattacharya and S. Roy, JHEP 0312, 015 (2003) hep-th/0309202. 
[13] P. K. Townsend and M. N. R. Wohlfarth, Phys. Rev. Lett. 91, 061302 (2003) hep-th/0303097].

[14] N. Ohta, Phys. Rev. Lett. 91, 061303 (2003) hep-th/0303238.

[15] S. Roy, Phys. Lett. B 567, 322 (2003) [hep-th/0304084].

[16] R. Emparan and J. Garriga, JHEP 0305, 028 (2003) [hep-th/0304124].

[17] C. -M. Chen, P. -M. Ho, I. P. Neupane, N. Ohta and J. E. Wang, JHEP 0310, 058 (2003) [hep-th/0306291].

[18] J. M. Maldacena and C. Nunez, Int. J. Mod. Phys. A 16, 822 (2001) hep-th/0007018].

[19] S. Kachru, R. Kallosh, A. D. Linde and S. P. Trivedi, Phys. Rev. D 68, 046005 (2003) hep-th/0301240].

[20] K. Dasgupta, G. Rajesh and S. Sethi, JHEP 9908, 023 (1999) [hep-th/9908088].

[21] S. B. Giddings, S. Kachru and J. Polchinski, Phys. Rev. D 66, 106006 (2002) hep-th/0105097].

[22] H. Bai, J. X. Lu and S. Roy, JHEP 0701, 094 (2007) hep-th/0610264.

[23] J. X. Lu, S. Roy, Z. -L. Wang and R. -J. Wu, Nucl. Phys. B 813, 259 (2009) arXiv:0710.5233 [hep-th]].

[24] N. Kaloper, J. March-Russell, G. D. Starkman and M. Trodden, Phys. Rev. Lett. 85, 928 (2000) hep-ph/0002001.

[25] G. D. Starkman, D. Stojkovic and M. Trodden, Phys. Rev. D 63, 103511 (2001) [hep-th/0012226].

[26] G. D. Starkman, D. Stojkovic and M. Trodden, Phys. Rev. Lett. 87, 231303 (2001) [hep-th/0106143].

[27] H. J. Boonstra, K. Skenderis and P. K. Townsend, JHEP 9901, 003 (1999) hep-th/9807137. 